Nouvelles perspectives en sciences sociales

\title{
Sur le thème de la complexité
}

\section{Claude Vautier}

Volume 4, numéro 2, avril 2009

Sur le thème de la complexité

URI : https://id.erudit.org/iderudit/029888ar

DOI : https://doi.org/10.7202/029888ar

Aller au sommaire du numéro

\section{Éditeur(s)}

Prise de parole

\section{ISSN}

1712-8307 (imprimé)

1918-7475 (numérique)

Découvrir la revue

Citer ce document

Vautier, C. (2009). Sur le thème de la complexité. Nouvelles perspectives en sciences sociales, 4(2), 9-11. https://doi.org/10.7202/029888ar d'utilisation que vous pouvez consulter en ligne.

https://apropos.erudit.org/fr/usagers/politique-dutilisation/ 


\section{Sur le thème de la complexité}

Depuis sa première parution, en 2005, la revue Nouvelles perspectives en sciences sociales (NPSS) a voulu, comme le
dit son énoncé de mission, réunir des approches se démarquant des entrées les plus courantes aujourd'hui en sciences sociales, qui sont, en sociologie notamment, les théories de l'action, le subjectivisme et, plus généralement, les théories du sujet inscrites dans une perspective phénoménologique. Ainsi que les textes publiés depuis quatre ans le montrent, ces positionnements n'ont pas été exclus de la revue, mais discutés, revisités, dans une perspective critique, mais aussi dans une inquiétude épistémologique conduisant aussi bien à s'interroger sur leurs fondements et leur légitimité que sur ceux des paradigmes concurrents proposés : aux analyses critiques des théories de l'action et de l'intentionnalité (vol. $1, \mathrm{n}^{\circ} 2$, vol. $2, \mathrm{n}^{\circ} 1$ ), aux propositions d'un paradigme relationnel (vol. $3, \mathrm{n}^{\circ} 1$, vol. $4, \mathrm{n}^{\circ} 1$ ), répondent des essais « transversaux " de modélisation par systèmes complexes interrogeant le discours et les représentations des acteurs (vol. 1, $\mathrm{n}^{\circ} 2$, vol. 2, $\mathrm{n}^{\circ} 1$, vol. 2 , n ${ }^{\circ} 2$, vol. $3, \mathrm{n}^{\circ} 2$, vol. $4, \mathrm{n}^{\circ} 1$ ), ou encore, dans le présent numéro, une réflexion sur les limites potentielles du systémisme complexe détaché de la question de l'intentionalité.

En ce sens, malgré un positionnement clairement critique des paradigmes dominants et orienté vers les paradigmes systémique complexe et relationnel émergents, NPSS s'est, dès l'origine, voulue une revue de débat dont l'objectif est fondamentalement 
de faire avancer la réflexion sur les modes de production de connaissances acceptables parce que pouvant être considérées comme relevant de critères de scientificité, certes eux-mêmes en discussion, mais cependant, en l'état, acceptables par une grande partie de la communauté scientifique.

Fidèle à cet esprit, considérant que le débat d'idées est la meilleure façon d'aborder de front les difficultés auxquelles se confrontent tous les chercheurs, inquiets de la qualité de leur production comme de leurs méthodes, le comité de direction a décidé d'ouvrir de façon systématique une nouvelle rubrique. Dans celle-ci, des chercheurs particulièrement reconnus pour leur contribution à un domaine ou à une approche sont invités à s'exprimer sur un thème différent dans chaque numéro. Les thèmes sont choisis en fonction de ce qui semble, selon le comité, être au cœur de la réflexion et des controverses actuelles ou parait le plus porteur de réflexions nouvelles et pertinentes pour l'ensemble du champ. Ces thèmes sont communs à un ensemble de sciences sociales (sociologie, certes, mais aussi urbanisme, géographie, économie, anthropologie, ethnologie, histoire, sciences politiques...) ou " naturelles ", comme la biologie, ou par nature " transversales ", comme l'informatique, sans doute plus difficile à classifier. Ils transcendent d'une certaine manière l'inter ou la transdisciplinarité revendiquée par le projet éditorial en se situant au niveau de questions telles que : "la complexité », « la relation", " la simulation ", indépendamment des objets qui peuvent être étudiés à partir de ces entrées.

Dans le présent numéro, c'est le thème de la complexité qui est traité par des auteurs bien connus et dont ni la compétence, ni la créativité, ni l'indépendance d'esprit ne peuvent être contestées : Henri Atlan, Jacques Lévy, pour l'aire européenne, David Krakauer et J. Stephen Lansing, pour l'aire anglo-américaine, Jacques Zylberberg l'aire franco-canadienne, disent ce que représente pour eux le vocable et sa mise en pratique dans les activités de recherche. Tous les auteurs pressentis n'ont pas répondu positivement, soit qu'ils n'en aient pas eu la possibilité matérielle, soit qu'ils aient jugé que le projet ne pouvait entrer 
dans leurs propres visées. Chez ceux qui ont accepté de contribuer, on trouvera des pensées divergentes, toujours talentueuses, pensons-nous (ce qui est cause de notre requête envers eux), certains étant des promoteurs convaincus du positionnement en débat, d'autres ses adversaires tout aussi déterminés, d'autres encore se situant dans une démarche de difficile synthèse.

Nous espérons que la rubrique qui s'ouvre avec cette livraison de Nouvelles perspectives en sciences sociales permettra de mettre en évidence les points focaux des désaccords, de désamorcer certains d'entre eux en éliminant les malentendus superficiels ou profonds et, sinon de concilier le peu conciliable, d'affirmer pour tous la légitimité de leur liberté à penser ce qu'ils pensent, étayée par leur honnêteté intellectuelle, épistémologique et méthodologique. Le débat scientifique, encombré par ses querelles d'ego, mais aussi ses impératifs institutionnels (la carrière, difficile et nécessaire poursuite d'un statut peu valorisé mais hors duquel un chercheur n'est pas réellement libre de chercher, de créer, de proposer, ne serait-ce que par manque de moyens) est trop souvent marqué au coin de l'acrimonie, de la stigmatisation, voire de l'insulte réciproque. Parmi les résultats attendus de cette nouvelle rubrique, et au-delà des résultats purement scientifiques, une pacification de ces relations serait aussi un succès important sur le plan de l'éthique scientifique. 